\title{
Factores de riesgo para COVID-19 en el personal de salud del Servicio de Emergencia del Hospital María Auxiliadora, marzo-diciembre 2020
}

Bernin Adderly Vera Cohaila* 1,a

\section{RESUMEN}

Objetivo: Determinar los factores de riesgo para presentar COVID-19 del personal de salud del Servicio de Emergencia del Hospital María Auxiliadora en el periodo marzo a diciembre de 2020.

Materiales y métodos: Investigación observacional, analítica-correlacional, transversal y retrospectiva. La muestra estuvo conformada por 117 trabajadores del Servicio de Emergencia. La técnica empleada fue la recolección de datos y el instrumento utilizado fue el cuestionario.

Resultados: El 25,64 \% de los participantes contrajo la COVID-19 por contacto con un familiar enfermo. Los médicos fueron el 38,49 \% y el 35,90\% realizaba labor asistencial en otra institución de salud. El 56,41 \% de los participantes trabajaba de 150 horas a más por semana; y 74,36 \% laboraba más de 12 horas por turno. Respecto al equipo de protección personal, el 78,64 \% percibió un uso correcto de este material, y el 17,90 \% lo usaba de forma incorrecta. Asimismo, el contacto con un familiar infectado por COVID-19 fue un factor de riesgo para infección por COVID-19 $(p=0,000 ;$ OR $=5,486)$.

Conclusiones: Los aspectos sociodemográficos y laborales no se evidenciaron como factores de riesgo para contraer la COVID-19. El factor patológico que sí demostró ser de riesgo fue el contacto con un familiar enfermo.

Palabras clave: Factores de riesgo; Personal de salud; Servicios médicos de urgencia; COVID-19 (Fuente: DeCS BIREME).

\section{COVID-19 risk factors among the healthcare personnel of the Emergency Department of the Hospital María Auxiliadora, March-December 2020}

\section{ABSTRACT}

Objective: To determine the COVID-19 risk factors among the healthcare personnel of the Emergency Department of the Hospital María Auxiliadora from March to December 2020.

Materials and methods: An observational, analytical-correlational, cross-sectional and retrospective research. The sample consisted of 117 emergency department workers. The study used surveys as technique and questionnaires as instrument for data collection.

Results: Twenty-five point six four percent $(25.64 \%)$ of the study participants contracted COVID-19 through a family member, $38.46 \%$ were doctors and $35.90 \%$ worked as medical practitioners in another health institution. Fifty-six point four one percent (56.41\%) worked 150 or more hours per week and $74.36 \%$ worked more than 12 hours per shift. Regarding the personal protective equipment, $\mathbf{7 8 . 6 3} \%$ wore it correctly while $17.90 \%$ used it incorrectly. Also, contact with a family member with COVID-19 was a risk factor for COVID-19 infection $(p=0.000, \mathrm{OR}=5.486)$.

Conclusions: The results indicate that sociodemographic and occupational aspects are not risk factors for COVID-19 infection. However, having contact with an infected family member proved to be a pathological risk factor.

Keywords: Risk factors; Health personnel; Emergency medical services; Coronavirus infections (Source: MeSH NLM).

1 Universidad Nacional Mayor de San Marcos. Lima, Perú.

a Médico internista.

*Autor corresponsal. 


\section{INTRODUCCIÓN}

El 2020 fue un año atípico en el panorama epidemiológico mundial debido a la aparición de un nuevo coronavirus que provoca una enfermedad viral que, en su desarrollo patogénico, presenta síntomas respiratorios que evolucionan rápidamente a un distrés severo que compromete la vida del enfermo ${ }^{(1)}$.

Al personal de salud le corresponde estar en la primera línea, frente a múltiples problemas (laborales, personales, sociales, psicológicos, de bioseguridad) y tratando de comprender los efectos y consecuencias de esta "nueva enfermedad" durante su jornada laboral, ya que la información cambia a un ritmo muy acelerado ${ }^{(1,2)}$. La Organización Mundial de la Salud (OMS) estimó que, hasta abril de 2020, más de 20000 trabajadores de la salud de 52 países habían contraído la COVID-19. En Italia, en ese momento, 15314 trabajadores de salud estaban infectados ${ }^{(3)}$; en México, en mayo, se reportaron 8544 enfermos (lo que corresponde al $23,5 \%$ de la población) (4). En Lima, Perú, se han registrado 6205 médicos con COVID-19, lo cual da una referencia a la cantidad total del personal de salud contagiada ${ }^{(5)}$.

Entre los problemas que debe de enfrentar cada trabajador sanitario están la alta exposición al virus, el riesgo elevado de contagio en relación a la población común, las medidas de contención implementadas en la entidad nosocomial, la disponibilidad de los equipos de protección personal (EPP), la calidad de los programas de vigilancia epidemiológica de cada país, entre otros ${ }^{(6,7)}$.

En el Servicio de Emergencia del Hospital María Auxiliadora, el personal de salud tiene una alta incidencia de contagios y un pequeño porcentaje de no contagio. Aunque comparten las mismas condiciones de exposición y bioseguridad, los residentes de Emergencias y Desastres tienen un promedio de contagio cercano al $70 \%$ (sin hospitalización); esta media es de $50 \%$ en el personal médico asistencial (tasa de hospitalización de casi $5 \%$ y una tasa de mortalidad del $3 \%$ ). El grupo más afectado, con un promedio de contagio aproximado de $80 \%$, es el personal de enfermería y los técnicos, con una media de hospitalización que está alrededor del $5 \%$, pero con mayor tasa de mortalidad y secuelas aproximada de $15 \%$.

El personal de salud afectado no precisa el momento en que la cadena de bioseguridad se interrumpió y se produjo la transmisión, o la reserva de los enfermos acerca de los factores precisos de contagio. Por lo tanto, es urgente dar mayor atención a la propagación de la enfermedad e identificar los factores de riesgo asociados a este proceso para establecer las medidas de control adecuadas y cuidar a este importante grupo. Por tal motivo, este estudio tiene como finalidad determinar los factores de riesgo para contraer COVID-19 en el personal de salud del Servicio de
Emergencia del Hospital María Auxiliadora, entre marzo y diciembre de 2020.

\section{MATERIALES Y METÓDOS}

\section{Diseño y población de estudio}

Investigación observacional, analítica correlacional, transversal y retroprospectiva desarrollada entre marzo y diciembre de 2020. La población de estudio estuvo conformada por 300 trabajadores de salud del Servicio de Emergencia del Hospital María Auxiliadora, mientras que la muestra fue seleccionada por conveniencia y agrupó a 117 trabajadores que cumplieron con los criterios de inclusión (personal que labore en el Servicio de Emergencia y que acepte participar en el estudio). El personal que estaba de vacaciones, con licencia, gestión o suspensión de actividades fue excluido del estudio.

\section{Variables y mediciones}

Las variables a analizar fueron las siguientes:

- COVID-19: Enfermedad causada por una cepa particular de la familia Coronaviridae que no había sido identificada antes en los seres humanos ${ }^{(8)}$.

- Factor de riesgo: Característica o rasgo de exposición que incrementa las probabilidades de padecer de una enfermedad (9).

- Factores sociodemográficos: Son todas las características asignadas a edad, sexo, educación, ingresos, estado civil, trabajo, religión, tasa de natalidad, tasa de mortalidad, tamaño de la familia. Esto se hace para cada miembro de la población ${ }^{(10)}$.

- Edad: Tiempo vivido por una persona expresado en años.

- Sexo: Condición orgánica, masculina o femenina.

- Personal de salud: Individuo que ejecuta actividades con la finalidad de promover la salud de la población en general ${ }^{(8)}$.

- Antecedentes de familiares con COVID-19: Familiar con COVID-19, lo que aumenta el riesgo en el paciente de padecer la enfermedad ${ }^{(11)}$.

- Comorbilidad en personal de salud: Presencia de enfermedades coexistentes o adicionales en relación al diagnóstico inicial.

- Contacto: Individuo expuesto de forma directa a un paciente probablemente infectado o con una prueba positiva ( 2 días previos o 14 días después del inicio de los síntomas) (12).

- Horas de trabajo: Cantidad de días de actividad laboral comprendidos en una semana o en un mes ${ }^{(13)}$.

- Procedimientos generadores de aerosoles: Situaciones que transmiten el COVID-19 por aire (ventilación mecánica con presión positiva, succión de las vías aéreas, intubación endotraqueal, fisioterapia torácica, broncoscopia, abordaje nebulizador e inducción de esputo) ${ }^{(14)}$.

- Equipo de protección personal: Elemento o dispositivo que incrementa la seguridad y preserva la salud del trabajador ante algún riesgo inminente de infección (15). 
Factores de riesgo para COVID-19 en el personal de salud del Servicio de Emergencia del Hospital María Auxiliadora, marzo-diciembre 2020

Para la recolección de datos empleamos las técnicas de la encuesta y la observación. Los instrumentos utilizados fueron el cuestionario y la lista de cotejo. El cuestionario fue autoadministrado y tenía respuestas semicerradas. La lista de cotejo verificó si el personal cumplía o no con el uso de EPP: el uso correcto implica que el trabajador utilizaba toda la indumentaria personal recomendada, según su actividad, durante toda la jornada laboral; mientras que se consideró el uso incorrecto si el profesional no utilizó al menos una indumentaria personal recomendada para su actividad laboral, o no la utilizó durante toda su jornada laboral.

\section{Análisis estadístico}

Para hallar la validez del instrumento se realizó una prueba piloto. La confiabilidad se evaluó mediante el método estadístico Kuder-Richardson y obtuvimos un valor de 0,81 que hace confiable al instrumento. La validez fue determinada por un juicio de expertos, en el que cinco profesionales con experiencia en el tema valoraron el cuestionario y dieron una concordancia del $95 \%$ entre los jueces. Para determinar los factores asociados para infección por COVID-19 se usó la prueba de ji al cuadrado y después se evaluó si dichos factores son de riesgo a través de Odds ratio, donde un $p$ valor menor a 0,05 fue considerado significativo.

\section{Consideraciones éticas}

Esta investigación se desarrolló bajo los lineamientos bioéticos de la Declaración de Helsinki ${ }^{(16)}$, dirigida a la investigación sanitaria. Asimismo, fueron considerados los cuatro principios bioéticos, es decir, la autonomía (por la que los pacientes tuvieron la libertad de decisión de participar o no en el estudio de investigación), beneficencia (este estudio proporciona mayor información científica sobre el riesgo de infección por COVID-19 en población sanitaria en el ámbito local y nacional), no maleficencia (la participación en el estudio no ocasionó riesgo alguno a su salud o integridad debido a que solo se aplicó un cuestionario, siguiendo todos los protocolos de bioseguridad implementados por la coyuntura actual) y justicia (todo el personal de salud que decidió participar en el estudio fue considerado sin realizar algún tipo de exclusión por posición social, económica, geográfica, etc.).

\section{RESULTADOS}

En el estudio participaron 117 trabajadores de salud del Servicio de Emergencia del Hospital María Auxiliadora: el $63,25 \%$ eran mujeres y el $51,28 \%$ estaba en el grupo etario de 25 a 34 años. El 20,51\% presentó alguna comorbilidad. El 25,64\% del personal evaluado presentó COVID-19. Los médicos fueron el $38,46 \%$, y el $21,37 \%$ no usaba correctamente el EPP.

No observamos ningún factor sociodemográfico asociado a la infección por COVID-19. Del grupo que tuvo la enfermedad, el 54,84 \% tenía entre 25 y 34 años; y el 25,81\%,35 y 44 años de edad (Tabla 1).

Tabla 1. Factores sociodemográficos del personal de salud del Servicio de Emergencia del Hospital María Auxiliadora (marzo-diciembre 2020)

\begin{tabular}{|c|c|c|c|c|c|}
\hline \multirow{3}{*}{$\begin{array}{c}\text { Factores } \\
\text { sociodemográficos }\end{array}$} & \multicolumn{4}{|c|}{ Infectado por COVID-19 } & \multirow{3}{*}{$p^{*}$} \\
\hline & \multicolumn{2}{|c|}{ Sí } & \multicolumn{2}{|c|}{ No } & \\
\hline & $\mathrm{N}$ & $\%$ & N & $\%$ & \\
\hline \multicolumn{6}{|l|}{ Sexo } \\
\hline Masculino & 13 & 41,94 & 30 & 34,90 & 0,485 \\
\hline Femenino & 18 & 58,06 & 56 & 65,10 & \\
\hline \multicolumn{6}{|l|}{ Edad (años) } \\
\hline$<25$ & 1 & 3,23 & 5 & 5,81 & - \\
\hline $25-34$ & 17 & 54,84 & 43 & 50,00 & 0,644 \\
\hline $35-44$ & 8 & 25,81 & 17 & 19,77 & 0,482 \\
\hline $45-54$ & 1 & 3,22 & 15 & 17,44 & 0,095 \\
\hline$>55$ & 4 & 12,90 & 6 & 6,98 & 0,312 \\
\hline Total & 31 & 100,00 & 86 & 100,00 & \\
\hline
\end{tabular}

* Prueba ji al cuadrado 
Estar infectado debido al contacto con algún familiar con COVID-19 se asocia de manera significativa a la infección COVID-19 $(p=0,000)$, con un factor de riesgo del 5,486 (Tabla 2): es decir, algún familiar que vive con el profesional de salud y que presente diagnóstico positivo para COVID-19 tiene cinco veces más probabilidades de contagiar, a diferencia del personal que no tiene familiares infectados en su vivienda. Además, se observó que el 51,61 \% de los enfermos tuvieron a un familiar infectado, en comparación con el 16,28\% de los que no se enfermaron (Figura 1).

Tabla 2. Factores patológicos del personal de salud del Servicio de Emergencia del Hospital María Auxiliadora (marzo-diciembre 2020)

\begin{tabular}{|c|c|c|c|c|c|c|}
\hline \multirow{3}{*}{$\begin{array}{c}\text { Factores } \\
\text { patológicos }\end{array}$} & \multicolumn{4}{|c|}{ Infectado por COVID - 19} & \multirow{3}{*}{$p^{*}$} & \multirow{3}{*}{$O R(I C)^{* *}$} \\
\hline & \multicolumn{2}{|c|}{ Sí } & \multicolumn{2}{|c|}{ No } & & \\
\hline & $\mathrm{N}$ & $\%$ & $\mathrm{~N}$ & $\%$ & & \\
\hline \multicolumn{7}{|c|}{ Tiene Ud. alguna comorbilidad } \\
\hline Sí & 7 & 22,58 & 17 & 19,77 & \multirow{3}{*}{0,739} & \\
\hline No & 24 & 77,42 & 69 & 80,23 & & 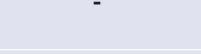 \\
\hline \multicolumn{5}{|c|}{ Contagiado de COVID-19 a } & & \\
\hline \multicolumn{7}{|c|}{ través un familiar } \\
\hline Sí & 16 & 51,61 & 14 & 16,28 & \multirow{3}{*}{\multicolumn{2}{|c|}{$\begin{array}{c}0,0005,486(2,213- \\
13,597)\end{array}$}} \\
\hline No & 15 & 48,39 & 72 & 83,72 & & \\
\hline Total & 31 & 100,00 & 86 & 100,00 & & \\
\hline
\end{tabular}

* Prueba ji al cuadrado// ** Odds ratio

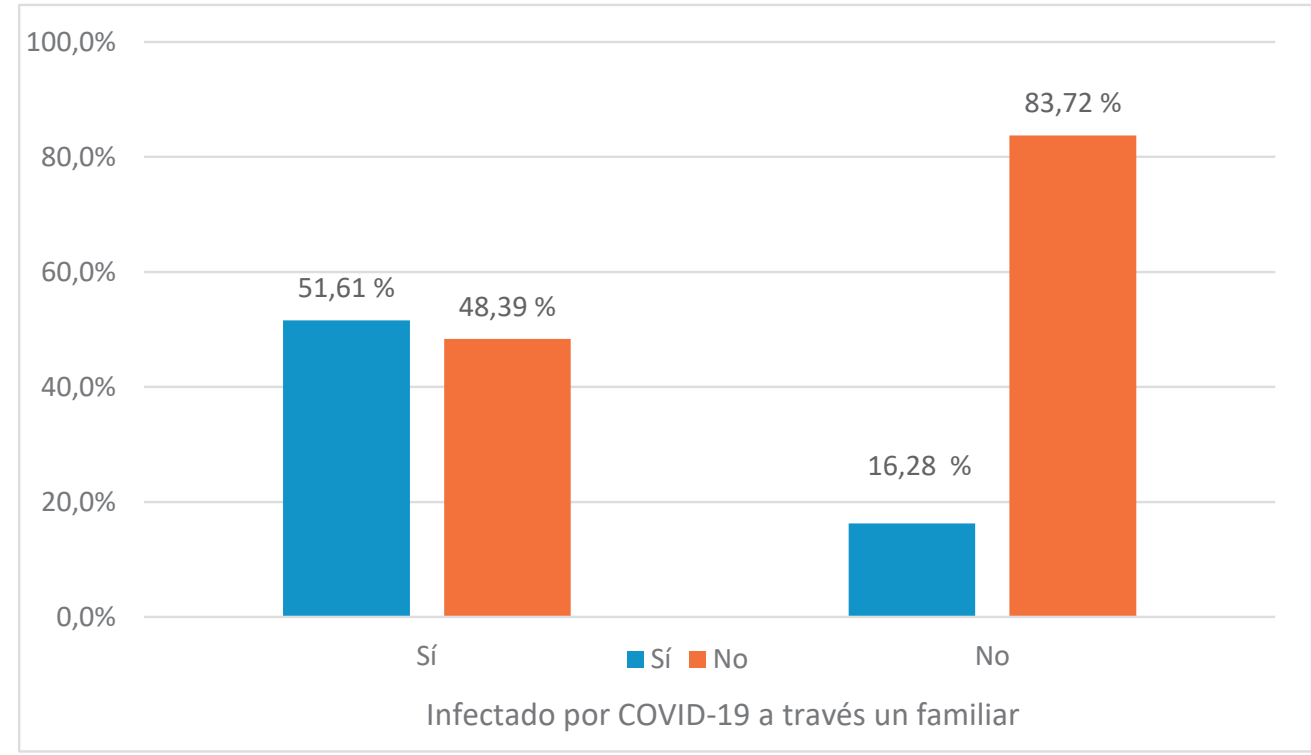

Figura 1. Infección por COVID-19 a través de una familiar

No se evidenciaron factores laborales asociados a la COVID-19, ya sea el tipo, labor asistencial, horas laborales al mes, horas laborales por turno, área de servicio y uso correcto de EPP $(p>0,05)$. Se observó que el $41,94 \%$ de los profesionales que se infectaron con COVID-19 fueron médicos, y que el 77,42 \% trabajaba en turnos de 12 horas a más (Tabla 3). 
Factores de riesgo para COVID-19 en el personal de salud del Servicio de Emergencia del Hospital María Auxiliadora, marzo-diciembre 2020

Tabla 3. Factores laborales del personal de salud del Servicio de Emergencia del Hospital María Auxiliadora, marzo-diciembre 2020

\begin{tabular}{|c|c|c|c|c|c|c|}
\hline & \multirow{3}{*}{$\begin{array}{l}\text { Factores } \\
\text { laborales }\end{array}$} & \multicolumn{4}{|c|}{ Infectado por COVID-19 } & \multirow{3}{*}{$p^{*}$} \\
\hline & & \multicolumn{2}{|c|}{ Sí } & \multicolumn{2}{|c|}{ No } & \\
\hline & & $\mathbf{N}$ & $\%$ & $\mathrm{~N}$ & $\%$ & \\
\hline \multicolumn{7}{|l|}{ Personal de salud } \\
\hline Medicina & & 13 & 41,94 & 32 & 37,21 & \multirow{7}{*}{0,712} \\
\hline Medicina interna & & 2 & 6,45 & 3 & 3,49 & \\
\hline Pediatría & & 0 & 0,00 & 4 & 4,65 & \\
\hline Traumatología & & 0 & 0,00 & 1 & 1,16 & \\
\hline Enfermería & & 9 & 29,03 & 27 & 31,40 & \\
\hline Técnico de enfermería & & 5 & 16,03 & 17 & 19,77 & \\
\hline Otro & & 2 & 6,45 & 2 & 2,32 & \\
\hline \multicolumn{6}{|c|}{ Realiza labor asistencial en otras instituciones } & \multirow{3}{*}{0,622} \\
\hline Sí & & 10 & 32,26 & 32 & 37,21 & \\
\hline No & & 21 & 67,74 & 54 & 62,79 & \\
\hline \multicolumn{6}{|c|}{ Horas laborales al mes en el hospital y otras instituciones } & \multirow{3}{*}{0,828} \\
\hline$<150$ & & 13 & 41,94 & 38 & 44,19 & \\
\hline$\geq 150$ & & 18 & 58,06 & 48 & 55,81 & \\
\hline \multicolumn{6}{|c|}{ Horas laborales realiza por turno } & \multirow{3}{*}{0,649} \\
\hline$\geq 12$ & & 24 & 77,42 & 63 & 73,26 & \\
\hline$<12$ & & 7 & 22,58 & 23 & 26,74 & \\
\hline \multicolumn{6}{|c|}{ En qué área del Servicio de Emergencia labora** } & \multirow{5}{*}{0,742} \\
\hline Triaje & & 1 & 3,22 & 3 & 3,49 & \\
\hline Tópico especializado & & 22 & 70,97 & 64 & 74,42 & \\
\hline Trauma shock & & 2 & 6,45 & 8 & 9,30 & \\
\hline Unidad crítica de emergencias & & 3 & 9,68 & 8 & 9,30 & \\
\hline \multicolumn{6}{|l|}{ Uso correcto de EPP } & \multirow[b]{3}{*}{0,353} \\
\hline Sí & & 24 & 77,42 & 68 & 79,07 & \\
\hline No & & 7 & 22,58 & 18 & 20,93 & \\
\hline \multicolumn{6}{|l|}{ Uso incorrecto de EPP } & \multirow{4}{*}{0,393} \\
\hline Sí & & 4 & 12,90 & 17 & 19,80 & \\
\hline No & & 27 & 87,10 & 69 & 80,20 & \\
\hline Total & & 31 & 100,00 & 86 & 100,00 & \\
\hline
\end{tabular}

* Prueba ji al cuadrado // **6 profesionales de salud no respondieron esta pregunta

\section{DISCUSIÓN}

Al realizar el análisis estadístico para identificar a los factores de riesgo para contraer COVID-19, no encontramos ninguna de las características sociodemográficas o laborales. Sin embargo, se halló, como único factor patológico, el estar enfermo por haber tenido contacto cercano con un familiar infectado $(p=0,000 ; \mathrm{OR}=5,486)$. Esto demostraría que las medidas de bioseguridad implementadas en el Servicio de Emergencia del Hospital María Auxiliadora son efectivas, considerando que los casos de contagio fueron externos al ámbito laboral. En consecuencia, la interacción social, sobre todo familiar, es una fuente permanente de contagio y de descuido en la interrelación de los miembros de la familia.

Al respecto, los estudios de Ran et al. ${ }^{(17)}(p<0,01, \mathrm{RR}=2,76)$, Celebi et al. ${ }^{(6)}(p=0,016)$ y Eyre et al. ${ }^{(18)}(p<0,001)$ también consideran que esta variable es un factor de riesgo para tener la COVID-19. Asimismo, Ran et al. ${ }^{(17)}(p<0,05, \mathrm{RR}=2,82)$ y Celebi et al. ${ }^{(6)}(p=0,04, \mathrm{OR}=11,295)$ concluyeron que el uso inadecuado de EPP es un factor de riesgo, lo que también ocurre en con la investigación de Nguyen et al. ${ }^{(19)}$ (ORa = 
1,49). Para Eyre et al. ${ }^{(18)}$, el no utilizar el EPP $(p<0,001)$ predispone a la aparición de la COVID-19, lo que coincide con lo reportado por Chatterjee et al. ${ }^{(20)}$, que considera esta variable como un factor de riesgo $(p<0,001, \mathrm{AOR}=5,33)$. Finalmente, Nguyen et al. ${ }^{(19)}$ señalan que las personas que reutilizaban las EPP tenían mayor probabilidad de contraer COVID-19 $(H R=5,06)$.

Nuestros resultados demuestran una realidad institucional en particular, lo cual podría considerarse como un elemento impulsor de otras investigaciones en el entorno nacional, para hallar otros factores relacionados a la COVID-19 en el personal sanitario. La identificación de estos factores es relevante para que las instituciones sanitarias puedan desarrollar otras estrategias que contribuyan a la reducción de los casos de COVID-19, lo que disminuiría no solo la morbilidad sino también la mortalidad en esta población que está en la primera línea de atención ante esta pandemia.

En esta investigación, el mayor número de enfermos de COVID-19 eran hombres, médicos, que tenían entre 25 y 34 años, y trabajaban en turnos de 12 horas a más. En conclusión, no encontramos evidencia de que las características sociodemográficas o laborales sean factores de riesgo para contraer COVID-19, pero sí observamos que el factor patológico asociado con la aparición de la enfermedad fue la cercanía a un familiar infectado.

Conflicto de intereses: Los autores declaran no tener conflictos de interés.

Financiamiento del articulo: El artículo ha sido financiado por los autores.

Contribuciones de los autores: Bernin Adderly Vera Cohaila fue el encargado de desarrollar, ejecutar y revisar el presente artículo de investigación.

\section{REFERENCIAS BIBLIOGRÁFICAS}

1. Muñoz-Fernández SI, Molina-Valdespino $\mathrm{D}$, Ochoa-Palacios $\mathrm{R}$, Sánchez-Guerrero 0, Esquivel-Acevedo JA. Estrés, respuestas emocionales, factores de riesgo, psicopatología y manejo del personal de salud durante la pandemia por COVID-19. Acta Pediatr Mex. 2020; 41(S1): 127-36.

2. Lozano-Vargas A. Impacto de la epidemia del Coronavirus (COVID-19) en la salud mental del personal de salud y en la población general de China. Rev Neuropsiquiatr. 2020; 83(1): 51-6.

3. Nienhaus A, Hod R. COVID-19 among Health Workers in Germany and Malaysia. Int J Environ Res Public Health. 2020; 17(13): 4881.

4. Del Carpio-Orantes L, Garcés-García E, Ortiz-Espinoza C, TorresSánchez JL, López-Varela LD, Pascual-Epigmenio S, et al. Medicos de primera línea de atención infectados por COVID-19 durante un brote hospitalario en Veracruz, México. Med Int Méx. 2020; 36(6): 781-8.

5. Paz D. Región con más contagios de COVID-19 en médicos [Internet] Diario La República: Lima; 02 de marzo del 2021. Disponible en: https: / / larepublica.pe/sociedad/2021/03/03/la-libertad-es-lasegunda-region-con-mas-contagios-covid-19-en-medicos-Irnd/ marzo.
6. Celebi G, Piskin N, Celik Beklevic A, Altunay Y, Salci Keles A, Ali Tuz $M$, et al. Specific risk factors for SARS-CoV-2 transmission among health care workers in a university hospital. Am J Infect Control. 2020; 48(10): 1225-30.

7. Poblete Umanzor R, Saldías Peñafiel F, Sabatini Ugarte N, Vite Valverde A, Ceriani Bravo A, Schaffeld Pernas S, et al. Infección respiratoria aguda por coronavirus Sars-CoV-2 en personal de salud. Implementación de un programa de deetcción precoz y seguimiento de casos en un hospital universitario. Rev Med Chile. 2020; 148(6): 724-33.

8. Organización Mundial de la Salud. Infecciones por coronavirus [Internet]. OMS; 2020. Disponible en: https://www.who.int/topics/ coronavirus_infections/es/

9. Organización Mundial de la Salud. Factores de riesgo [Internet]. OMS; 2020. Disponible en: https://www.who.int/topics/risk_factors/es/

10. Organización Panamericana de la Salud. Descriptores en Ciencias de la Salud - Factores socioeconómicos [Internet]. OPS; 2020. Disponible en: http://decs2020.bvsalud.org/cgi-bin/wxis1660.exe/decsserver/

11. Ministerio de Salud. Norma técnica de salud para el uso de los equipos de protección perosnal por los trabajadores de las instituciones prestadoras de servicios de salud. Norma Técnica de Salud $N^{\circ} 161$ MINSA/2020/DGAIN [Internet]. MINSA: Lima; 2020. Disponible en: https://www.gob.pe/institucion/minsa/normas-legales/783241. 456-2020-minsa

12. Grishaw J. COVID-19: Definiciones de casos. 1a ed.: McGraw-Hill Education Inc; 2020.

13. Organización Panamericana de la Salud. Descriptores en Ciencias de la Salud - Horas de trabajo [Internet]. OPS; 2020. Disponible en: http://decs2020.bvsalud.org/cgi-bin/wxis1660.exe/decsserver/.

14. Organización Panamericana de la Salud. 2020: Alertas y actualizaciones epidemiológicas [Internet]. PAHO; 2020. Disponible en: $\quad$ https: / / www3.paho.org/hq/index.php?option=com docman\&view=list\&slug=2020-alertas-epidemiologicas\&ltemid=270\& layout=default\&lang=es

15. Ministerio de Salud y Protección Social. Programa de elementos de proteccion personal, uso y mantenimiento [Internet]. MINSALUD: Colombia; 2017. Disponible en: https://www.minsalud.gov.co/ Ministerio/Institucional/Procesos\%20y\%20procedimientos/GTHS02.pdf

16. Mazzanti D, Ruggiero MA. Declaración de Helsinki, principios y valores bioéticos en juego en la investigación médica con seres humanos. Rev Colomb Bioet. 2011; 6(1): 125-44.

17. Ran L, Chen X, Wang Y, Wu W, Zhang L, Tan X. Risk factors of healthcare workers with coronavirus disease 2019: a retrospective cohort study in a designated Hospital of Wuhan in China. Clin Infect Dis. 2020; 71(16): 2218-21.

18. Eyre DW, Lumley SF, O'Donnell D, Campbell M, Sims E, Lawn E, et al. Differential occupational risk to healthcare workers from SARS CoV-2: A prospective observational study. Elife. 2020; 9: e60675.

19. Nguyen LH, Drew DA, Joshi AD, Guo C-G, Ma W, Mehta RS, et al. Risk of COVID-19 among front-line health-care workers and the general community: a prospective cohort study. medRxiv. 2020.

20. Chatterjee P, Anand T, Singh KJ, Rasaily R, Singh R, Das S, et al. Healthcare workers \& SARS-CoV-2 infection in India: A case-control investigation in the time of COVID-19. Indian J Med Res. 2020; 151(5): 459-67. 
Factores de riesgo para COVID-19 en el personal de salud del Servicio de Emergencia del Hospital María Auxiliadora, marzo-diciembre 2020

\section{Correspondencia:}

Bernin Adderly Vera Cohaila

Dirección: Calle José G. Paredes 130 -A. Pueblo Libre, Perú.

Teléfono: +51 947994564

Correo electrónico: bernin.vera@hotmail.com

Recibido: 10 de marzo de 2021

Evaluado: 23 de abril de 2021

Aprobado: 04 de mayo de 2021

(c) La revista. Publicado por Universidad de San Martín de Porres, Perú.

(c) Br Licencia de Creative Commons Artículo en acceso abierto bajo términos de Licencia Creative Commons Atribución 4.0 Internacional. (http://creativecommons.org/licenses/by/4.0/)

ORCID iDs

Bernin Adderly Vera Cohaila i https://orcid.org/0000-0002-0209-4587 